\title{
Procesos educativos a distancia: hacia la construcción de procesos integrales e inclusivos en el Ecuador
}

\section{Distance Education Processes: Towards the Construction of Integral and Inclusive Processes in Ecuador}

\author{
Giancarlo Giorgio De Agostini ${ }^{1 *}{\text { Frank } \text { Viteri }^{2} \text { y Marco Yamba-Yugsi }}^{3}$ \\ ${ }^{1}$ Universidad Católica de Cuenca \\ 2 Universidad Central del Ecuador \\ 3 CEC-IAEN \\ *giancarlo.deagostini@ucacue.edu.ec
}

DOI: https://doi.org/10.26871/killkana_social.v3i2.420

\begin{abstract}
Resumen
A lo largo del presente trabajo, se realiza un análisis de los procesos educativos a distancia desde la contextualización de dichos procesos, la finalidad es atender a las necesidades de las personas beneficiarias, pero también analizar las categorías de calidad, justicia social y pertinencia en el sistema de educación a distancia, lo cual evitará la homogeneización de la educación a distancia, ya que de hacerlo, no podrá atender a todos los grupos vulnerables y excluidos. El llamado es repensar estos procesos educativos, no como una extensión del sistema formal - presencial, sino como una modalidad independiente que responde a las necesidades contextualizadas. Y que aunque se haga uso de categorías de la educación presencial, estas deben ser adecuadas a las particularidades de la población a la que se dirige, caso contrario, se constituiría en un margen de la educación presencial.
\end{abstract}

Palabras clave: educación a distancia, calidad, pertinencia, justicia social, contextualización, homogeneización.

\begin{abstract}
Throughout this paper, an analysis of distance education processes is carried out based on the contextualization of these processes. The purpose of this research is to meet the beneficiaries' needs, and to analyze quality categories, social justice, and relevance of the distance education system; this will prevent the homogenization of distance education since, if this happens, this system will not be able to reach all vulnerable and excluded groups. The call is made to reconsider these educational processes, not as an extension of the formal face-to-face system, but instead as an independent modality that responds to contextualized needs. Although categories of face-to-face education are been used, they must be adapted to the particularities of the population to which they are aimed. Otherwise, this would become a limitation of face-to-face education.
\end{abstract}

Key words: distance education, quality, relevance, social justice, contextualization, and homogenization.

\section{Introducción}

En el presente trabajo, se pretende analizar la importancia de la contextualización en los procesos educativos a distancia, para establecer procesos que dignifiquen a la persona en su totalidad. Por ser distintos necesitan una atención especial, en el sentido que deben ser entendidos desde las prácticas pedagógicas en base a una fundamentación legal, teórica y epistemológica que viene establecida desde la Autoridad Educativa Nacional, como una base en la cual se puedan desarrollar las iniciativas fiscales o particulares. En este sentido, se trata de poder cumplir con estándares establecidos, de manera contextualizada y no como una extensión o un margen ${ }^{1}$ de la educación formal presencial.

El proceso educativo ha evolucionado a lo largo de la historia generando cambios sustanciales en el mismo, así como también propuestas que respondan a las necesidades de cada una de las épocas socio-históricas que el hombre ha

\footnotetext{
${ }^{1}$ En los actuales modelos educativos muchas de las iniciativas de educación a distancia, o para adultos ha tenido que desaparecer por no poder ajustarse a los requerimientos fiscales ya que se imponen los mismos procesos de la educación formal presencial, sean: procesos administrativos, evaluativos, de acreditación, metodológicos, didácticos, etc. Lo cual ha generado que estas iniciativas sean debilitadas por no cumplir con los estándares de la educación formal - presencial, lo cual es obvio por sus características diferentes.
} 
ido atravesando. Ahora bien, en la actualidad los problemas humanos y sociales han ido en aumento, provocando que la educación busque nuevas alternativas para llegar a todas las personas. A finales del siglo XIX e inicios del XX aparecen nuevas alternativas de educación a las cuales se las llamarán, educación a distancia, se refieren a: "modalidades de enseñanza que recaen, total o parcialmente, en alguien que no comparte el mismo tiempo y espacio que el alumno ${ }^{2}$, y que tiene como misión alcanzar una mayor apertura y flexibilidad en la educación, ya sea en términos de acceso, programas de estudio u otros aspectos de su estructura" (UNESCO, 2003, p.13). Luego pasaran a constituirse en grandes programas de educación desde las iniciativas estatales o particulares con el fin de llevar una educación a los grupos vulnerables y excluidos.

\section{Caracterización teórica de la Educación a Distancia}

Una de las características importantes del sistema educativo a distancia es la flexibilidad. Esta es importante en el mundo contemporáneo, ya que por medio de la educación formal - presencial no se ha logrado llegar a todas las personas que constituyen el conglomerado social, o muchas de ellas han sido excluidas de este sistema educativo. Por sus condiciones de vulnerabilidad quienes no han podido culminar sus estudios, las ofertas educativas a distancia tendrán como fundamento principal, democratizar el acceso a la educación, ya que ha demostrado ser una forma de organizar propuestas de estudio sumamente flexibles, y ha posibilitado el acceso a la educación a sectores diversos y tradicionalmente excluidos del sistema convencional (Coicaud, 2002).

Pero el imperativo no es solo el llevar educación a grupos excluidos, sino también, establecer nuevas alternativas para constituir una educación para todos, que puedan desenvolverse en la sociedad del conocimiento que día a día va constituyéndose en un escenario cambiante con el fin de desarrollar el pensamiento crítico y estimular la actitud científica desde la primera escuela y a lo largo de toda su vida educativa (Gomez Buendía, 1998). Esto va a permitir al ciudadano que ingrese en los procesos educativos, dar soluciones a los inconvenientes que vaya a encontrar en su vida. Pues como explica Bauman: si se ve a la educación como un proceso, esta debe estar en un continuo crecimiento, en continuas búsquedas de interpretar y reinterpretar esta realidad, (...), ya que este está en continuo cambio, de la misma manera en la que un software se actualiza, la educación debe estar en una constante evolución. (Bauman, 2007). La educación y en especial los procesos de enseñanza-aprendizaje no pueden estar supeditados a cuatro paredes de cemento y ladrillo.

El problema que genera la falta de acceso a los procesos educativos sobre todos los grupos excluidos y vulnerables, se ha constituido en una preocupación constante

\footnotetext{
${ }^{2}$ Tiempo y espacio que comparten directamente estudiantes y docentes en los sistemas de educación formal presencial, en cualquiera de los niveles y subniveles educativos.
}

en los estados y la sociedad civil con el fin de emprender actividades conjuntas para llegar a todos con una educación de calidad. De ahí que en la Declaración Mundial sobre Educación para Todos, de la UNESCO, hace un llamado a buscar opciones que disminuyan los efectos de este problema, lo cual reza así en su artículo primero:

Cada persona -niño, joven o adulto- deberá estar en condiciones de beneficiarse de las oportunidades educacionales ofrecidas para satisfacer sus necesidades básicas de aprendizaje. Estas necesidades comprenden tanto las herramientas esenciales para el aprendizaje (tales como lectura y escritura, expresión oral, aritmética, resolución de problemas) como los contenidos básicos mismos del aprendizaje (conocimientos teóricos y prácticos, valores y actitudes) requeridos para que los seres humanos sean capaces de sobrevivir, desarrollen sus capacidades, vivan y trabajen con dignidad, participen plenamente en el desarrollo, mejoren la calidad de sus vidas, tomen decisiones fundamentadas y continúen aprendiendo. El alcance de las necesidades básicas de aprendizaje y la manera de satisfacerlas varía según cada país y cada cultura e, inevitablemente, cambia con el paso del tiempo (UNESCO, 1990, p.7).

En estas condiciones el llamado a la estructuración de procesos educativos flexibles que lleguen a la mayor cantidad de personas desde el estado como garante y proveedor de servicios básicos, para que sus ciudadanos logren romper con aquellos modelos pedagógicos opresivos que han venido dejando de lado, a un gran conglomerado social, (Freire, 2005), y otro imperativo de carácter universal en el cual debe constituirse en agentes que propicien una educación para todos desde sus contextos. Al decir de Vivas Herrera, conviene entender que los componentes que permiten asegurar la optimización de la calidad educativa, continuamente se ponen bajo la lupa, es decir, están siendo evaluados entre ellos, para avanzar hacia una mejor educación para todos. (...) ya que las sociedades modernas cambian y por consiguiente exige de todos mejorar para optimizar la calidad educativa (Vivas Herrera, 2012).

En este marco, surge la Educación a Distancia como una herramienta fundamental para permitir el acceso a la educación de todas las personas, no solo por la masificación del proceso educativo, sino por las facilidades y la ampliación de la población beneficiada, cumpliendo con el llamado humano de permitir el acceso a la educación a toda la población mundial: derecho fundamental y reconocido por todos. Dewey expresa que "la educación de adultos es simultáneamente un derecho y un bien público al que todos deberían tener acceso, pero en la que todos tienen igualmente la responsabilidad de participar, en el interés de construir y mantener la democracia" (Dewey, 1900).

Este preámbulo surge debido a que no se da el cumplimiento de dichas declaraciones, que muchas veces han quedado en manifiestos, memorias, actas y tratados, pero 
no han encontrado asidero en la realidad, sobre todo por la falta de recursos económicos y consensos para fomentar políticas locales que permitan una apertura del proceso educativo a toda la humanidad, desde la contextualización de las iniciativas que pueden surgir desde la realidad. De ahí que se pretende analizar la modalidad de educación a distancia como una herramienta que permita llegar con una educación de calidad y pertinente a la mayoría de personas que quedaron fuera del proceso educativo.

\section{Necesidad de un enfoque praxeológico de la educa- ción a distancia, desde los contextos}

Es importante partir de una definición de educación a distancia: "cualquier proceso educativo en el que toda o la mayor parte de la enseñanza es llevada a cabo por alguien que no comparte el mismo tiempo y/o espacio que el alumno, por lo cual toda o la mayor parte de la comunicación entre profesores y alumnos se desarrolla a través de un medio artificial, sea electrónico o impreso" (UNESCO, 2003, p.27), este canal de comunicación distinto y flexible es el que permite un mayor campo de acción a las personas para acceder al proceso educativo, debido a que no es necesario tener un espacio físico común en el cual establecerse para que se dé la praxis educativa.

No se trata de delimitar etimológica o conceptualmente a la educación a distancia, o de cerrar el marco de acción que esta debe tener, es decir, anulando la flexibilidad propia de esta modalidad. Sino establecer las bases en las que se debe sostener este proceso, de ahí que "la naturaleza "abierta" de la educación que utiliza los medios electrónicos o impresos como vehículo, puede institucionalizarse de modo formal en el contexto de políticas como la admisión abierta y la libertad de elección de qué, cuándo y dónde aprender" (UNESCO, 2003, p.28).

El presente trabajo de igual manera considera importante la fundamentación teórica no solo del proceso sino también de la educación para adultos, a la cual la educación a distancia ha contribuido.

Se conoce como Educación para Adultos a la totalidad de los procesos organizados de educación, sea cual sea el contenido, el nivel o el método, sean formales o no formales, ya sea que prolonguen o reemplacen la educación inicial dispensada en las escuelas y universidades, y en forma de aprendizaje profesional, gracias a las cuales las personas consideradas como adultos por la sociedad a la que pertenecen, desarrollan sus aptitudes, enriquecen sus conocimientos, mejoran sus competencias técnicas o profesionales o les den una nueva orientación, y hagan evolucionar sus actitudes o su comportamiento en la doble perspectiva de un enriquecimiento integral del hombre y una participación en un desarrollo socioeconómico y cultural equilibrado e independiente (UNESCO, 1976).

Definiciones que serán analizadas en el marco de la pertinencia, calidad y justicia social, como las bases de esta estructura, pero conviene entender que el Estado se debe constituir en el garante de estos procesos y modelos, ya que es esta "institución la que concretiza el acuerdo social de un pueblo, pues es un actor importante dentro de la elaboración de políticas para la educación y dentro del debate sobre las dimensiones filosóficas sobre las cuales se quiere construir el proceso educativo" (Reyes, 2009), y es este quien regulará las iniciativas tanto públicas como privadas, a favor de estos procesos educativos, siempre en el marco del diálogo, ya que estas experiencias no son comunes y tienen características propias que deben ser explicadas, analizadas, dialogadas y comprendidas desde ambas partes, pero siempre con una sustentación epistemológica clara y así evitar una homogeneización de la educación a distancia. Ya que esta exige:

La participación política, ya sea como iniciativa gubernamental o por parte de organizaciones internacionales, es necesaria para el desarrollo y futuro de esta modalidad. Pero una política basada en acciones integradoras no solo demanda la iniciativa del Estado, sino que también la sociedad civil y las organizaciones internacionales las cuales deben tomar parte activa del proceso (OEI, 2009).

Es muy difícil llegar a un consenso claro sobre la definición de este modelo, pero se puede estructurar una base de acción con unos objetivos definidos de modo muy claro a lo largo del hecho educativo como tal. El papel de la Autoridad Educativa Nacional en el campo de la educación a distancia es fundamental para establecer políticas claras de base; donde no se cierren las puertas sino que en base al análisis y diálogo se pueda establecer una práctica educativa abierta bien sustentada, lo cual se evidencia con claridad en el Informe Mundial sobre el Aprendizaje y la Educación de Adultos:
Al reafirmar la centralidad del derecho a la edu- cación y las competencias básicas a lo largo de toda la vida teniendo a la alfabetización como piedra angular de este derecho, la Declaración de Hamburgo enfatizo la responsabilidad del Estado en la provisión y financiación de una educación de adultos de calidad. Atrajo la atención hacia la necesidad de alianzas entre el Estado, la sociedad civil y el sector privado en el desarrollo y el sostenimiento del aprendizaje y la educación de adultos (UNESCO, 2010, p.26). En este sentido instituciones como la Fundación Virtual Iberoame- ricana creada en el Ecuador en el 2002 creó el Colegio Virtual Iberoamericano con aprobación del MINEDUC en el 2004, institución que ofrece los últimos tres años de la Escuela Básica $\left(8^{\circ}, 9^{\circ}\right.$, $\left.10^{\circ}\right)$ y los tres años de Bachillerato $\left(1^{\circ}, 2^{\circ}, 3^{\circ}\right)$ para así graduarse.

Para ampliar la definición de educación a distancia, con el apoyo de las tecnologías digitales de avanzada, se ofrece la siguiente definición como aprendizaje en línea:

"La Educación a Distancia virtual o aprendizaje en línea (e-Learning) es un proceso de enseñanzaaprendizaje colaborativo, basado en trabajos colectivos e individuales, esencialmente con actividades 
motivadoras, colaborativas y participativas asíncronas, mediado por las nuevas tecnologías para el aprendizaje y el conocimiento (nTAC) con apoyo de recursos interactivos, síncronos y asíncronos, con contenidos multimedia, para así crear una comunidad de aprendizaje que genera compromiso y conocimiento, constructivamente", 3 .

A partir de la comprensión de modelos educativos alternativos y su fundamentación establecida desde el Estado, se puede dar apertura a prácticas educativas abiertas y a distancia que ayuden a paliar los problemas de rezago en educación en constante cooperación entre Estado y actores sociales. De forma tal que no se establezcan políticas que cierran el campo de acción de la modalidad, ni anulen la flexibilidad de la misma sino que establezcan fundamentos claros de la Educación a distancia, sin la cual no puedan actuar quienes hacen uso de esta modalidad. Lo anterior se plantea con el fin de dar la apertura necesaria a nuevas iniciativas, pero garantizando el cumplimiento de los objetivos educativos, en la mesa de diálogo con las iniciativas sociales para evitar la homogeneización del sistema educativo a distancia, ya que la inclusión no puede ser homogénea sino mas bien contextualizada. Por ejemplo, no pueden darse los mismos procesos educativos a distancia a las personas privadas de la libertad como a las personas beneficiarias de las aulas hospitalarias, cada una de estas poblaciones tienen características propias que deben ser tomadas en cuenta al

${ }^{3}$ De Agostini, Giancarlo. (2004) momento de ofertar estos modelos educativos. Deben estar fundamentadas en políticas públicas establecidas en base a:

- Calidad

- Justicia Social

- Pertinencia

Estos tres fundamentos, se encontrarán enraizados a manera de pirámide de modo que el proceso educativo no se pueda dar sin uno de estos (teniendo como eje a la persona), ya que anularían la complementariedad del mismo, y no se establecería un proceso de humanización y dignificación de la persona. Figura 1

Desde el tratamiento integral de esta visión de la Educación a distancia, es conveniente establecer una correlación entre cada uno de estos componentes que permiten desarrollar un proceso de educación a distancia holística garantizada desde las políticas públicas y fundamentadas en las prácticas particulares.

\section{Fundamentación de los procesos educativos a dis- tancia desde los parámetros de calidad}

Los procesos educativos a distancia deben estar fundamentados en el principio de calidad educativa, que sobre todo en el Ecuador, que desde la Constitución, Plan del Buen Vivir, Plan Decenal de Educación, etc., ha sido asumida como presupuesto fundamental de todo el sistema público, fiscomisional y particular de educación sin distinción de modalidades:

(... ) un concepto de calidad educativa complejo y multidimensional, según el cual el sistema educati-

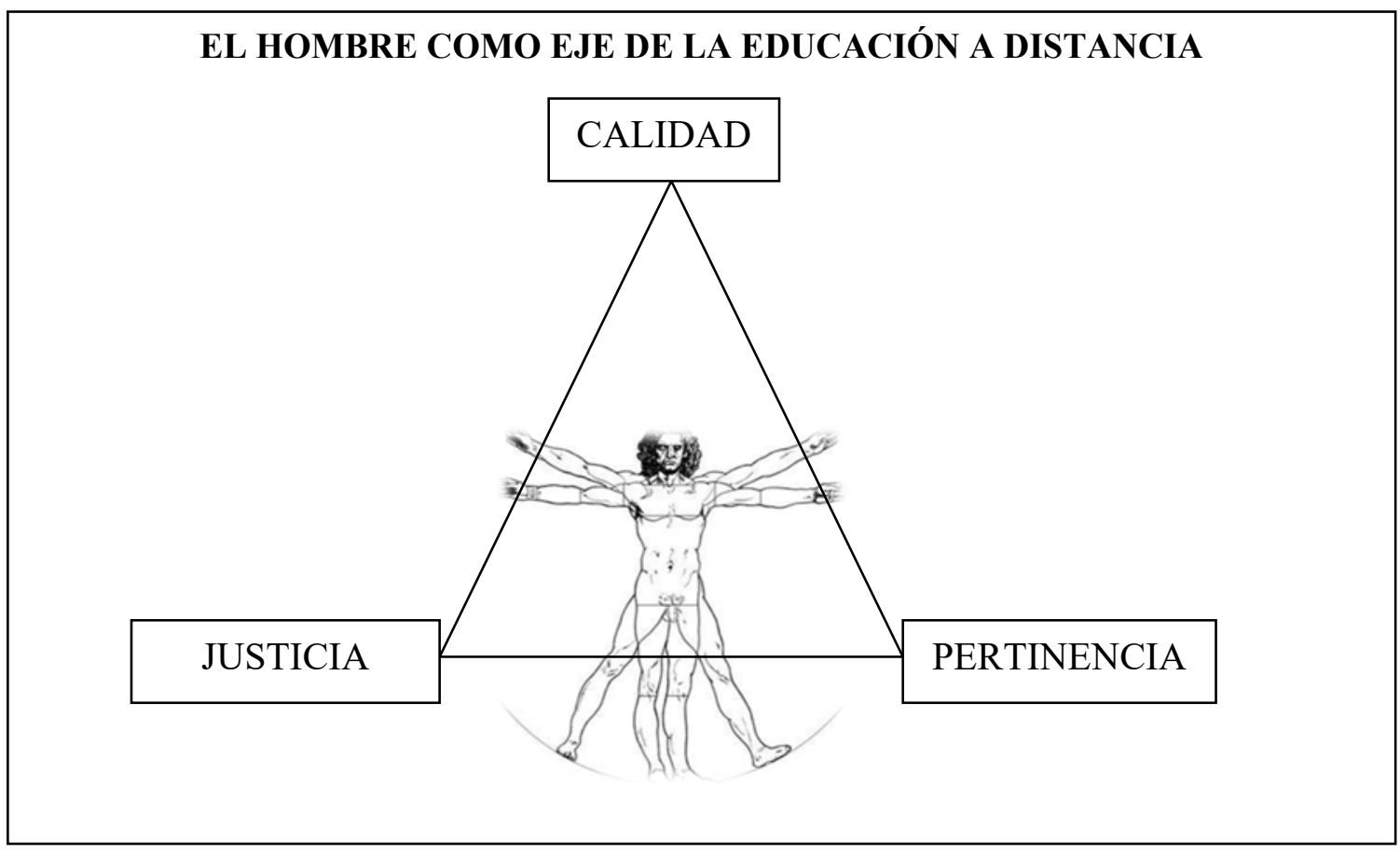

Figura 1. La fundamentación de los procesos educativos a distancia desde: calidad, pertinencia y justicia social con el fin de contribuir a la humanización de las personas excluidas 
vo será de calidad en la medida en que los servicios que ofrece, los actores que lo impulsan y los productos que genera contribuyan a alcanzar ciertas metas o ideales conducentes a un tipo de sociedad democrática, armónica, intercultural, próspera, y con igualdad de oportunidades para todos (Ministerio de Educación del Ecuador, 2010).

Desde estos presupuestos la Educación a distancia no debe concebirse como un modelo que termina efímeramente con los vacíos y rezagos de los sistemas educativos formales y por lo tanto pueden ser tomados a la ligera. De ahí que en el Reglamento a la Ley Orgánica de Educación Intercultural, en su artículo 24 dice:

La modalidad de Educación a distancia debe cumplir con los mismos estándares y exigencia académica de la educación presencial. Para la promoción de un grado o curso al siguiente, y para la obtención de certificados y títulos, los estudiantes que se educan mediante esta modalidad deben certificar haber adquirido los aprendizajes mínimos requeridos del grado o curso en un examen nacional estandarizado, según la normativa que para el efecto emita el Nivel Central de la Autoridad Educativa Nacional (Asamblea Nacional del Ecuador, 2012).

Aunque los parámetros de calidad estén establecidos en las leyes, muchas veces, estos parámetros son los mismos que la educación formal, lo cual se convierte en un problema, pues las características no son las mismas, por lo tanto, los resultados de evaluación de estos parámetros no pueden ser satisfactorios o a su vez pueden constituirse en una falsedad. Los parámetros deben ser contextualizados para que se garantice un resultado claro y objetivo, abriendo paso a la intervención del Estado con el fin de mejorar con carácter permanente, por lo que el cumplimiento de los estándares establecidos por la Autoridad Educativa Nacional, deben ser contextualizados desde la realidad de la educación para adultos, para no convertir a la educación a distancia en una extensión de parámetros de la educación formal presencial.

Aunque las categorías que se usen para explicar este fenómeno educativo, sean los mismos de la educación formal - presencial: promociones, certificaciones, evaluaciones, exámenes, etc., el proceso debe ser incrustado en la propuesta educativa particular, desde la realidad de la población a la que se atiende, por lo que "se trata de repensar y redefinir la pertinencia sociocultural y política de la educación, de lo que se enseña y se aprende (saberes y epistemologías) y de cómo se enseña y se aprende (métodos y recursos (...))" (Villagomez y Cunha de Campos, 2014, p.39). Ya que se debe caminar hacia la deshomogenización del establecimiento de políticas en la educación para adultos, y desde la contextualización caminar hacia la capacidad para diseñar, ejecutar, canalizar, evaluar, programas que combinan directrices claras con oportunidades para adap- tarse flexiblemente a las necesidades y condiciones locales para lograr un currículo de calidad (UNESCO, 2010).

No se puede establecer solo un reconocimiento como tal de esta actividad educativa, sino generar políticas públicas que garanticen una Educación a distancia de calidad para que las personas que acceden a ella no solo se puedan reinsertar o ascender en el campo laboral, sino también acceder a sistemas de educación superior con sólidas destrezas conceptuales, teóricas y actitudinales que les permitan cumplir con los retos universitarios, de ahí que se ha de tener en cuenta las exigencias de la sociedad del conocimiento lo que conlleva el reconocimiento, a través del sistema de homologación de créditos, de las competencias y las calificaciones (Consejo de la Unión Europea, 2002).

\subsection{Hacia una justicia social, desde la Educación a dis- tancia}

Al revisar la literatura sobre el nacimiento de la educación a distancia sea por: correspondencia, apoyo tecnológico, virtual, se evidencia que esta nace para llegar a los excluidos de la educación formal presencial, a aquellos grupos vulnerables que por distintas razones han pasado a formar parte del grupo de los rezagados de la educación. Pues bien, la educación a distancia debe tener un carácter claro de justicia social con la cual se atiende a los excluidos del ámbito educativo, pero no se atiende como un acto de piedad a los grupos vulnerables, de ahí que los otros dos factores de la pirámide, permiten a la educación a distancia constituirla como un acto humanizador, ya que la sociedad necesita educar a las personas para que se constituyan en agentes productivos que contribuyan a una sociedad más justa, desde la justicia con la que son tratados. En este aspecto en el Ecuador se establecen políticas claras, pero a la vez contradictorias entre sí, pues en unas se hacen llamados a contextualización mientras que en otras se establece la homogenización de la educación, sin tomar en cuenta factores particulares:

Las ofertas de educación para personas con escolaridad inconclusa brindan la oportunidad de concluir los estudios en los diferentes niveles y sub niveles educativos a los jóvenes y adultos que son parte de los grupos vulnerables y excluidos del sistema educativo y del modelo económico, social y político. Proponen procesos educativos de calidad, no muy largos, de utilidad y aplicación inmediata, que preparan para la vida futura. Responden a la heterogeneidad de los estudiantes y a sus diversos contextos, con currículos y propuestas pedagógicas diversificadas y servicios educativos adecuados a sus necesidades y particularidades (Ministerio de Educación, 2012).

Aunque se establecen modelos curriculares diversos y alternativos, siempre existirán factores normativos que cierran las puertas a estas modalidades, sobre todo en los procesos de evaluación y acreditación, donde no se toma 
en cuenta las características de los grupos vulnerables y excluidos, lo anterior, convierte a la educación a distancia, en una extensión de la educación formal y por lo tanto deben ser acreditados y evaluados de igual manera, de acuerdo a la normativa y a los procesos. Muchas veces porque se toman los parámetros de calidad, desde la medición y comparación numérica con otras épocas o con otros contextos, cuando la calidad debe también ser vista desde las realidades en las que se aplica, antes que recurrir solo a una comparación numérica y estadística.

En el ámbito de la justicia social de la educación a distancia, todos los actores de la sociedad debe comprender que este hecho debe ser constituido desde perspectivas distintas a la educación presencial, pero fundamentada en principios de calidad, ya que "las barreras que el aprendizaje a distancia tal vez logre derrumbar incluyen no solo la distancia geográfica, sino también otros obstáculos, tales como limitaciones personales, barreras culturales y sociales y la falta de infraestructura educativa" (UNESCO, 2003, p.25). De ahí que la categoría de justicia social debe estar fundamentada en el principio de calidad propios que legitimen el hecho educativo como tal, tomando en cuenta que "la inclusión digital es un componente integral de la inclusión social en un mundo cada vez más influenciado por las tecnologías de la información y las comunicaciones. Las TIC pueden y deben ser usadas como una herramienta efectiva en la lucha contra el hambre, la pobreza y el analfabetismo" (Conferencia Regional Ministerial de América Látina y el Caribe, 2005) . No como acto de piedad sino como restitución del derecho a la educación a los grupos excluidos, de ahí que es fundamental:

\begin{abstract}
"desarrollar programas académicos virtuales y a distancia de calidad, basados en un modelo pedagógico sólido, con una amplia gama de herramientas TAC y a precios accesibles es fundamental; especialmente aquellos dirigidos a sectores de escasos recursos económicos, académicamente vulnerables y con menor acceso a la tecnología por los costos" (De Agostini, 2013)
\end{abstract}

En base a esto se deben establecer metas concretas, que permitan integrar la apertura de iniciativas que surjan del uso de las nuevas tecnologías para poder llegar a las grandes multitudes, que por sus condiciones, sociales, familiares, laborales y económicas no pueden acceder a un sistema educativo presencial. Si se establecieron objetivos reales y con características propias que permitan cumplir los objetivos concretos, colegios y universidades como FUVIA con el Colegio Virtual Iberoamericano (Ecuador), UNED (España), UTPL (Ecuador), iniciativas que usan los medios electrónicos para llegar con una educación de calidad para todos aquellos que por distintas características no pueden estar en la modalidad presencial en una escuela, colegio y universidad. "Donde el reto, siempre será encontrar vías para acreditar los resultados relacionados con el desarrollo y el crecimiento personal, y mantener la flexibilidad en los sistemas de acreditación y evaluación". (UNESCO, 2010, p.83)

\subsection{Necesidad de modelos educativos pertinentes}

La educación se constituye en una herramienta que permite el ascenso en las escalas sociales, de manera que se garantiza la movilidad social, con el fin de mejorar las condiciones de vida las personas y sus familias. Ahora bien, la educación a distancia debe ser pertinente de acuerdo a las condiciones sociales, por lo que se debe definir en un primer momento a la pertinencia lo que significa que el "aprendizaje en los programas debe representar una vía eficaz y apoyo para la transformación personal y social, una fuente para mejorar la calidad de vida" (UNESCO, 2010, p.80). Ya que las personas que recurren a una educación a distancia, lo hacen porque no pudieron, en su época o situación, acceder a sistemas educativos formales.

Pero como fenómeno de la época contemporánea cada vez aparecen condiciones que no permiten a las personas continuar con sus estudios en la edad establecida, asociadas o no a la discapacidad. Dejando de lado perspectivas de la cultura dominante en estos procesos educativos, no se pueden estructurar estos procesos como una extensión de la educación formal presencial, sino mas bien propiciar "la elaboración del diseño educativo y el contenido del aprendizaje, sustentados en el conocimiento y los valores endógenos para asegurar la supervivencia de la identidad cultural en una economía global crecientemente impulsada por la tecnología" (Ahmed, 2009). La contextualización del proceso educativo generará no solo un cambio en la situación socio - económica del beneficiario sino también conservará un bagaje cultural local.

Al aumentar los niveles de deserción y abandono de los procesos educativos formales presenciales, los sistemas educativos a distancia deben surgir de la realidad de las poblaciones a las que se van a atender, desde un análisis situacional que permita establecer de forma clara objetivos ¿Qué se debe enseñar?, ¿cómo?, ¿para qué?, ¿cómo evaluar?, ¿cuándo evaluar?, preguntas que deben ser respondidas por los garantes de una educación de calidad, pues la población y sus características no son las mismas de la educación formal. Ya que "la educación de adultos, por el contrario, busca aportar beneficios inmediatos y palpables en la calidad de vida de los educandos; de no ser así, su participación podría disminuir" (UNESCO, 2010, p.81).

De ahí, que la Autoridad Educativa Nacional, debe establecer una agenda con fines claros, de lo que se desea consolidar con la educación a distancia, ya que no se puede trabajar con los mismos fines, cambiando las metodologías o la frecuencia de la presencialidad, muchas personas que acceden al sistema educativo para adultos, no necesariamente busca continuar con estudios superiores de tercer o cuarto nivel, aunque el ideal fuese ese. Sino que esta educación busca mejorar las condiciones de vida de cada uno de los asistentes, ya que muchos de los beneficiarios de estos procesos educativos abandonan los mismos porque 
ven que los procesos educativos a distancia "tienen poca pertinencia para mejorar la propia vida y las perspectivas de empleo. Si bien las barreras actitudinales son de naturaleza sociopsicológica, también están entrelazadas con las barreras institucionales y situacionales, e interactúan con ellas" (UNESCO, 2010, p.80).

Por lo que requieren currículos, planificaciones, procesos administrativos y material pedagógico en específico concebido para educandos distintos, que están abiertos y se basan en diversas fuentes de información y son capaces de adaptarse a las realidades locales y a diferentes grupos de destinatarios (UNESCO, Informe mundial sobre el aprendizaje y la educación de adultos, 2010); de manera que las agendas se constituyan de una cooperación interinstitucional desde distintos sectores como: productividad, industrias, agricultura, salud, empleo, inclusión económica y social, telecomunicaciones, cultura, turismo etc., para estructurar objetivos, que de cumplirse, directamente mejoren la calidad de vida de las personas, ya que cuando la educación se relaciona con la transformación económica, actúa como un catalizador de la alfabetización de adultos y la educación básica en derechos humanos (Schmelkes, 1990).

Cuando no se cumplen con las expectativas directas de los educandos adultos, el fracaso es directo en el cumplimiento de los objetivos, por lo que "la motivación depende de la oferta de un contenido pertinente que tenga en cuenta el contexto y las expectativas de los educandos adultos. Su participación en la definición y el diseño de los programas de educación de adultos puede contribuir a mejorar su autoestima personal y cultural (UNESCO, 2010).

En este sentido se considera que una fundación ecuatoriana ${ }^{4}$ cumple bastante con estas metas utilizando con éxito las TAC $^{5}$ desde el 2004 con resolución n $^{\circ} 3507$ del MINEDUC.

Para finalizar este acápite unas palabras de Nicholas Negroponte (1995), todavía muy vigentes hoy día: (libre traducción) "Posiblemente lo que ocurre hoy en nuestra sociedad no son alumnos incapaces de aprender, pero más bien ambientes educativos incapaces de enseñar. La tecnología en apoyo a la educación, bien utilizada, cambia esta situación, permitiéndonos alcanzar a un alumnado con diferentes estilos cognitivos y pedagógicos."

\subsection{Hacia la construcción de procesos educativos a dis- tancia como formas de educación integral}

Los procesos educativos a distancia se constituyen en alternativas muy convenientes para establecer una educación permanente, sin estereotipos de educación en etapas de la niñez y adolescencia. Las personas que fueron excluidas del sistema educativo presencial formal, pueden encontrar una alternativa de superación humana y profesional en

\footnotetext{
${ }^{4}$ Fundación Virtual Iberoamericana (FUVIA) creadora del Colegio Virtual Iberoamericano (CVI), totalmente "en línea".

${ }^{5}$ TAC: Tecnologías del Aprendizaje y el Conocimiento. De Agostini, Giancarlo, 2004
}

la adultez, de ahí que "las nuevas formas de entender la educación conciben al ser humano en un continuo «hacerse». Unas estructuras flexible ayudarán al individuo a desenvolverse en un proceso de formación continua y de perfeccionamiento permanente" (Sarrate y Pérez de Guzman, 2005, p.44).

Entonces se rompe con la idea de que estos procesos educativos se constituyen en meros actos de piedad para los grupos excluidos o a su vez para personas que no cumplen con los requerimientos mínimos para ingresar a la educación formal presencial. De ahí que se deben establecer entre los lineamientos bases de este proceso educativo, categorías de: calidad, justicia social y pertinencia, de manera que se logre cumplir con el objetivo de educar de una manera integral.

Aunque el repensar estos procesos exigen ejercicios de contextualización de iniciativas, la invitación se mantiene siempre desde el Estado y la sociedad civil, ya que siempre van a existir grupos excluidos y vulnerables que no puedan acceder a los procesos educativos en el "momento y lugar determinados por la cultura". Este es el camino, como dice la UNESCO, de generar una educación para todos, respetando el derecho del hombre a la educación. Por lo que la educación a distancia debe hoy y siempre caminar hacia una educación incluyente, pero diferenciada, donde el objetivo principal sea la formación integral del individuo desde el desarrollo de aptitudes, la adquisición valores y de conocimientos, mejorando las competencias técnicas y profesionales (...) desarrollando todas sus potencialidades, que inciden en especial en la dimensión social para así posibilitar la participación del adulto en la expansión socioeconómica y sociocultural" (Sarrate y Pérez de Guzman, 2005) del País.

En los últimos tiempos los estados trabajan muy arduo por generar este tipo de procesos educativos, deben entender que no pueden homogeneizar los mismos, ya que la vulnerabilidad y la exclusión no es la misma para todos. La amplia gama de ofertas educativas a distancia, presenciales, semipresenciales o totalmente "en línea", deben estar a disposición de las necesidades de cada uno de los futuros beneficiarios. Por lo que las políticas públicas deben ser fruto del diálogo entre todos los actores para que no se cierren las oportunidades de estudiar a pesar de sus condiciones.

Así como también los procesos de acreditación, evaluación, estructuración de currículos, metodologías y didácticas deben ser contextualizadas a las necesidades de cada una de las personas a ser incluidas y no solo ver a estos procesos como extensión de la educación formal para paliar problemas sociales. De que el repensarlos de una manera académica, sustentándola científicamente y entendiendo las iniciativas diferenciadas será el llamado a todos los actores de la educación a distancia para reivindicarla como un proceso de formación integral, dejando de lado prácticas de poca calidad que no responden a los fines propios de la educación sea cual sea la modalidad. 


\section{Referencias Bibliográficas}

Ahmed, M. (2009). The State and Development of Adult Learning and Education in Asia and the Pacific: Regional Synthesis Report (Inf. Téc.). Hamburgo.

Asamblea Nacional del Ecuador. (2012). Reglamento General a la Ley Orgánica de Educación Intercultural. Quito: Registro Oficial de la República del Ecuador.

Bauman, Z. (2007). Los retos de la educación en la modernidad líquida. Barcelona: Gedisa.

Coicaud, S. (2002). La democratización del conocimiento a trávez de la educación a distancia.Étic@ net. ISSN: $1695-324 X, 20-33$.

Conferencia Regional Ministerial de América Látina y el Caribe. (2005). Compromiso de Río de Janeiro (Inf. Téc.). Río de janeiro.

Consejo de la Unión Europea. (2002). Resolución del Consejo de la Unión Europea de 27 de junio de 2002 sobre la educación permanente. Bruselas: Diario Oficial de las Comunidades Europeas.

De Agostini, G. (2013). El significado de una praxis para la educación en linea: El aspecto psicológico de la motivación. Sophia: Colección de Filosofía de la Educación, 187-216.

Dewey, J. (1900). La escuela y la sociedad. Chicago: University Chicago Press.

Gomez Buendía, H. (1998). La Agenda Del Siglo XXI: Hacia un Desarrollo Humano. T/M Editores: México.

Ministerio de Educación. (2012). Ministerio de Educación. Descargado de http://educacion.gob.ec/ educacion-para-jovenes-y-adultos/

OEI. (2009). Concepción y tendencias de la educacion a distancia en América Látina. Madrid: Centro de Altos Estudios Universitarios de la OEI.

Sarrate, L., y Pérez de Guzman, V. (2005). Educación de personas adultas: situación actual y perspectivas de futuro. Revista de Educación - UNED, 41-57.

Schmelkes, S. (1990). Postalfabetizacion y trabajo en América Látina. México: CREFAL/UNESCO -
CRELAC.

UNESCO. (1976). Actas de la Conferencia General. En Recomendación relativa al desarrollo de la educación de adultos. contenido de la educación. resolución 19 (p. 124). Paris: Autor.

UNESCO. (1990). Declaracion Mundial sobre Educación para Todos . En Conferencia mundial sobre educación para todos (pp. 1-42). Jomtien: Autor.

UNESCO. (2003). Aprendizaje abierto y a distancia (Inf. Téc.). Paris.

UNESCO. (2010). Informe mundial sobre el aprendizaje y la educación de adultos (Inf. Téc.). Ginebra.

Villagomez, M. S., y Cunha de Campos, R. (2014). Buen Vivir y educación para la práctica de la interculturalidad en el Ecuador. Otras prácticas son necesarias. . Revista Alteridad - Universidad Politecnica Salesiana, 34-42.

Vivas Herrera, J. (2012). Educar y conocimiento: El problema del conocer en la escuela. Sophia - Universidad Politecnica Salesiana, 232-250.

Recibido: 28 de febrero de 2019

Aceptado: 26 de julio de 2019 